\title{
Case Files of the Medical Toxicology Fellowship Training Program at the New York City Poison Control Center: Hypotensive Death-Therapeutic Complication or Suicide?
}

\author{
S. Eliza Halcomb, MD, Lewis S. Nelson, MD
}

New York City Poison Control Center, New York, NY

\section{CASE PRESENTATION}

A 55-year-old patient with a history of hypertension, hepatitis C, and depression arrived at an urgent care center complaining of abdominal discomfort and vomiting. Her systolic blood pressure was $70 \mathrm{mmHg}$ and her heart rate was 55 beats per minute. The patient was transferred to a local Emergency Department (ED) for evaluation. On arrival, she was awake, alert, and appropriate. Her vital signs included a blood pressure of $88 / 50 \mathrm{mmHg}$, a heart rate of 48 beats per minute, and a respiratory rate of 16 breaths per minute. She was afebrile. Her physical examination was otherwise unremarkable. A finger-stick showed a blood glucose concentration of $190 \mathrm{mg} / \mathrm{dL}$.

Further history revealed that the patient started taking clonidine, $0.1 \mathrm{mg}$ bid two days prior to presentation. After her third dose of clonidine, she noticed abdominal discomfort. Her other medications included atenolol, $50 \mathrm{mg}$ bid and sustained-release diltiazem, $360 \mathrm{mg}$ daily. She denied suicidal ingestion and continued to complain only of abdominal discomfort.

An ECG showed third-degree heart block. Pacemaker pads were placed, and 50 grams of oral activated charcoal was administered. IV access was obtained and $2 \mathrm{~L}$ normal saline was administered, resulting in a mild improvement of her blood pressure.

Laboratory tests revealed a metabolic acidosis with a $\mathrm{pH}$ of 7.12, $\mathrm{pCO}_{2} 53 \mathrm{mmHg}, \mathrm{pO}_{2} 63 \mathrm{mmHg}$, and serum bicarbonate $16 \mathrm{mEq} / \mathrm{L}$. The patient's arterial lactate was $6.9 \mathrm{mmol} / \mathrm{L}$, and a chemistry profile was remarkable for bicarbonate of $16 \mathrm{mEq} / \mathrm{L}$, BUN $20 \mathrm{mg} / \mathrm{dL}$ and for creatinine of $2.1 \mathrm{mg} / \mathrm{dL}$.

The patient was initially treated with 1 gram of calcium chloride and had transient improvement of her blood pressure.
Within an hour after presentation, the patient became pulseless and unresponsive. She was resuscitated with $1 \mathrm{mg}$ of epinephrine and $1 \mathrm{mg}$ atropine, both administered by intravenous bolus. Infusions of atropine and glucagon (dosages unclear) were also initiated. Her blood pressure improved to $110 / 70 \mathrm{mmHg}$, and her heart rate rose to 60 beats per minute. The regional poison center was subsequently contacted, and the center recommended discontinuation of the atropine infusion, bolus dosing of calcium chloride 1 gram (13.5 mEq of calcium) as needed, up to 5 grams initially, as well as the addition of a high-dose insulin and dextrose infusion. Strong consideration for pulmonary artery pressure monitoring was suggested. The treating physician felt uncomfortable with this regimen and opted instead to add a norepinephrine infusion to the patient's original treatment regimen. Over the next hour, the patient became increasingly bradycardic and hypotensive. In spite of maximal treatment with calcium, atropine, and norepinephrine-she expired.

\section{Which cases are required by law to be reported to the medical examiner?}

At the present time, there is no federal law that mandates reporting deaths to the medical examiner. Every jurisdiction has its own requirements. It is advisable to contact local authorities to determine which cases require reports to the medical examiner (ME). Deaths that should generally be referred to the ME include but are not limited to:

Deaths caused by homicide, suicide or accidental deaths from overdoses, falls, and motor vehicle crashes.

Unusual or unexpected deaths-including suspected foul play.

Keywords: bradycardia, calcium channel antagonists, diltiazem Corresponding Author: S. Eliza Halcomb, MD, New York City Poison Control Center, $4551^{\text {st }}$ Avenue, Room 123, New York, NY 10016. Email: halcoe01@med.nyu.edu 
Deaths caused by an agent or disease thought to be a threat to public health.

Death related to a medical procedure.

Deaths that occur in the workplace.

Deaths that occur in institutionalized patients or people in custody [1].

\section{How is the manner of death determined?}

The medicolegal determination of death has a rich and colorful history. The role of the coroner was established by the English in $1194 \mathrm{AD}$. The word coroner is derived from the word crown, and the position signified that the coroner was an agent of the king. At that time the determination of the manner of death was of great importance to the crown because it had economic implications. Felony offenses, such as murder, resulted in the levying of fines against the perpetrator and the village in which the death took place. Suicide was also considered a felony, and the decedent's property was turned over to the royal treasury. Needless to say, there was limited medical knowledge, and a tremendous amount of political pressure accompanied the office of the coroner. The position had minimal credibility, as it was beholden to political influences.

In the American colonies, autopsies were prohibited, with an exception granted for teaching medical students. The first recorded autopsy in a criminal investigation occurred in Maryland in 1665. Although the victim in this case was noted to have sustained a skull fracture and intracranial hemorrhage, the coroner suggested that the patient had died "because he had not gone to a doctor" [paraphrased] and the defendant was declared innocent [2]. Later, as coroners were compensated for the number of deaths that they certified, it purportedly became common practice to discover a body (e.g., in a river), certify the death, and return the body to its initial location of discovery (the river) to be "rediscovered" at a later time. As a result of such acts, by the late 1800 s the office had lost all credibility [2]. The state of Massachusetts abolished the office of coroner in 1877. New York City followed suit in 1915, and the Office of the Medical Examiner was established to create a more scientific determination of the causes of deaths [3]. In 1918, the term "manner of death" was included on death certificates and included the following choices: natural, accident, suicide, homicide and indeterminate [3].

Currently, coroners in the U.S. are elected laypeople responsible for conducting investigations of deaths [4]. Up to $25 \%$ of the population in this country is covered by a coroner system that has no requirement for medical expertise or death investigation experience $[4,5]$. There is also a great deal of interstate variability in training programs for coroners, and this can greatly impede the quality of death investigations [4]. To address this issue, many regions have replaced the coroner system with the Office of the Medical Examiner. However, this trend has been slow to develop, with less than half of the US population, in 1997, being served by medical examiner systems [6]. Medical examiners are physicians (often pathologists) who are officially appointed to serve a city, county or region. Neither MEs nor coroners are required to have experience in forensics, and less than 100 physicians have become board-certified in forensic medicine since 1959 , so there is a wide variation in the quality of death investigations across the US $[7,6]$.

\section{What does the medical examiner do when a case is referred?}

Once the ME has determined the need to review a case, there is often a scene investigation that involves documenting the circumstances of the death, as well as the patient's medical, social, and psychiatric history. Many decedents undergo autopsy, although this is not a requirement. Based on evidence and opinion, the ME assigns a "cause of death" (a medical determination) as well as a "manner of death" (a medico-legal determination). But the determination of the cause and manner of death is not always straightforward. Medical examiners rely on their training and education as well as their investigative staff (forensic toxicologist) and the medical literature to arrive at conclusions regarding cause and manner of death.

The pronouncement of a manner of death by a medical examiner is occasionally the source of public and private controversy. The importance of this determination is noted in the fact that religious burial or life insurance payments, to list but a few examples, are often linked to this determination. Considerable inconsistencies in determining the manner of death exist between individual MEs and coroners. A recent survey of 198 medical examiner and coroners evaluated the differences in manner of death classifications for twenty-three representative scenarios. There was only one scenario with $100 \%$ agreement among the subjects, and only 11 cases with $>80 \%$ agreement. Sixty three percent $(63 \%)$ of the participants also felt that the term "therapeutic complication" should be added to the list of choices, and some felt that the classification, manner of death, should be removed from the death certificate altogether. The paper recommended the establishment of national guidelines to achieve better consistency between medical examiners [8]. This trend toward the use of national guidelines to reduce variability in medical care echoes the lexis of the general movement in much of medicine.

Similarly, there is an occasional disagreement between the ME and another party linked to the decedent, such as the physicians who cared for the patient prior to death. The other party may be or may believe to be directly impacted by the determined manner of death. For example, the treating physician may perceive that their liability for the patient's death hinges on the medical examiner's determined manner of death. This sentiment is supported by documented information on a death certificate that is generally considered definitive.

Although the medical examiner is the final arbiter of the manner of death, the court, on occasion, may be asked to decide. A review of lawsuits that challenged the manner of death found that in 15 of 16 evaluated cases, a lower court upheld the forensic findings. However, in 5 of those 15 , the decisions were later 
reversed by a higher court. For example, one challenge was brought by the parents, both physicians, of an infant whose manner of death was ruled a homicide. They provided convincing evidence from multiple experts that the death was due to natural causes, and the Ohio Supreme Court ordered a change to the manner of death on the death certificate [9].

Several well publicized deaths of people in police custody have received considerable attention. An ME's investigation may be of critical importance to the public and to prison officials. If an ME determines that the cause of death is natural, there may be a widely publicized outcry of bias or influence from the family and friends of the deceased. According to some authors, MEs may be pressured by officials to offer a favorable opinion [2]. Historic and recent examples of this are noted in the literature: A former Chief Medical Examiner of New York City was allegedly fired for repeatedly refusing to change the cause of death on certificates [2]. After attributing the death of several teenage girls killed in a car crash to inhalant abuse, a Pennsylvania Medical Examiner was released from duties following a public disagreement with the parents of the victims [10]. The Pennsylvania ME reportedly reconstructed the scene by spraying the inhalant in a car and measuring his own blood concentrations of the product, and he concluded that the blood concentrations in the victims were high enough to prove abuse rather than ambient exposure [10].

\section{CASE CONTINUATION}

Since the circumstances surrounding the case were uncertain, the body was sent to the medical examiner's office for postmortem evaluation. A scene investigation at the patient's home revealed no evidence of drug stockpiling, and the diltiazem pill container had its full contents. The patient's physician was interviewed, and the physician stated that the patient was also on fluoxetine for depression, although the bottles were never found, and the patient had not revealed this information to the emergency physician.

A postmortem examination was unrevealing. No pills were detected in the stomach or gastrointestinal tract. Analysis of the postmortem heart blood revealed an atenolol concentration slightly above the therapeutic range and a therapeutic clonidine concentration. The diltiazem concentration was $13,900 \mathrm{mcg} / \mathrm{L}$ (therapeutic range: $86-188 \mathrm{mcg} / \mathrm{L}$ ). The cause of death was determined to be cardiotoxicity complicating diltiazem administration. The Medical Examiner determined that the manner of death was due to therapeutic complication.

\section{Why was the manner of death in this case considered a therapeutic complication rather than a suicide?}

Once again, the cause of death is a medical term. Given the blood concentration of diltiazem and the complete clinical picture, it is clear that the patient's cause of death is diltiazem toxicity. The manner of death, however, is a medico-legal term based on evidence and opinion. Although the clinical and laboratory features of this case are consistent with an acute overdose, there is not any scene evidence to support this decision. Although the patient had a history of depression, a high blood concentration of diltiazem, and all of the clinical features of calcium channel antagonist poisoning, the patient apparently denied a history of overdose. The patient's bottles of medication purportedly contained accurate pill counts. Additionally, blood analysis confirmed that the patient took multiple therapeutic medications and analysis confirmed that the medications were not taken in overdose. Despite the impressive blood concentrations of diltiazem, there was insufficient forensic evidence to establish suicide as the cause of death.

A reasonable argument could be made that the high blood diltiazem concentration suggests that the patient took an acute, intentional overdose. The approximate fatal blood concentration reported in the literature is $15,000 \mathrm{mcg} / \mathrm{L}$, similar to the concentration in this patient [11]. However, diltiazem blood concentrations may vary 10-fold following a standard dose [12]. Diltiazem redistribution occurs postmortem from the tissue, leading to elevated concentrations in heart blood, which was the site of the sampling in this case. Basic drugs with large volumes of distribution tend to accumulate in the lungs and diffuse postmortem into the right and left heart chambers, suggesting that high concentrations of diltiazem in heart blood postmortem have actually redistributed from the lungs rather than the liver or heart [13]. Furthermore, postmortem blood concentrations may be misleading since the lysis of erythrocytes (which accumulate diltiazem) following death may contribute to substantially higher concentrations than would be expected during premortem therapeutic dosing.

\section{Is the death certificate ever wrong? What are the potential pitfalls in defining manner of death?}

A recent pilot study looked at the quality assurance process in a medical examiner's office over a one-year period. The ME certified 1267 deaths and 47 (approximately 4\%) of the certificates had erroneous information. Of these 47 incorrect certificates, two (approximately 4\%) listed an erroneous cause of death and three (approximately 6\%) suggested an incorrect manner of death. All of the certificates were amended. To explain the incorrect determination of the cause or manner of death, the study noted the lack of timely results of forensic toxicology testing as one of the key factors [14].

Another review examined the pitfalls of attributing the manner of death to suicide when, in fact, there may be a drug-drug interaction or altered pharmacokinetics. In patients with hepatic insufficiency, there is a $69 \%$ increase in oral diltiazem bioavailability [12]. An oral dose of $360 \mathrm{mg}$ of diltiazem administered to a patient with liver failure, in whom there is markedly reduced first-pass elimination, may conceptually raise a patient's serum concentrations above the therapeutic range and produce cardiotoxicity. Thus, the bioaccumulation of the drug-even at therapeutic dosing - may lead to the erroneous assumption that 
the toxic blood concentration is due to an acute overdose rather than to a therapeutic complication [15]. Although this patient did not have liver failure, it is unclear to what magnitude the blood concentration of diltiazem may rise following therapeutic administration in a patient with liver failure and whether the precipitous onset of clinical toxicity is consistent with a therapeutic misadventure.

Although it is attractive to estimate the dose of a drug based on blood concentrations, it is difficult to extrapolate theoretical premortem doses from postmortem drug concentrations because the volume of distribution in an individual patient may vary and postmortem redistribution may elevate blood concentrations. One gets an analytically true elevation but a clinically false positive.

The presence of a drug in blood postmortem should not necessarily suggest that the drug is related to the cause of death. For example, the National Association of Medical Examiners guidelines on certifying cocaine-related deaths commented that there are three possible prerequisites for determining a cocaine related death: the history surrounding the death can be associated with acute cocaine exposure, or; the immediate cause of death is readily identifiable and associated with cocaine, or; chronic cocaine use leads to an ultimately fatal disease process [16].

Another study from forensic toxicology literature addressed the potential for errors in death investigations due to a relative unfamiliarity with clinical pharmacology. The study suggested that most physicians are continually learning about pharmacology because they are actively prescribing drugs. This is often not the case for forensic pathologists and death investigators. This characterization, however, may be an overly generous statement about practicing clinicians because there is variability in the degree to which all medical personnel maintain timeliness of their information. The study identified the most commonly encountered pharmaceuticals in death investigations and called for forensic physicians to use pharmacology texts to familiarize themselves with the mechanism of actions of these agents [17]. Interestingly, the author of the study did not mention the role of the medical toxicologist in assisting the medical examiner.

Medical toxicologists can play a substantial consultative role in the postmortem assessment of the toxicologic cause of death. Medical toxicologists can assist in the interpretation of postmortem drug data and provide a medical examiner with premortem and postmortem pharmacokinetic and clinical insight. The role of a medical toxicologist in assisting in the determination of the manner of death is less clear, but medical toxicologist may, for example, provide insight into typical workplace conditions, therapeutic dosing regimens, or substance abuse demographics.

\section{Which pharmacologic agents could be responsible for this patient's presentation?}

Clonidine should be included in the differential diagnosis for hypotension and bradycardia. Clonidine is an imidazoline with potent $\alpha_{2}$-adrenergic receptor agonism. Central stimulation of these receptors results in presynaptic inhibition of sympathetic tone, leading to a decrease in blood pressure and heart rate $[18,19]$. Similarly, stimulation of central imidazoline receptors results in lowering of blood pressure [20]. Other clinical manifestations of clonidine toxicity include CNS depression, miosis, and hypoventilation that mimic opioid toxicity and are occasionally reversible with naloxone.

Atenolol is a $\beta$-adrenergic antagonist that primarily exerts its hypotensive effects by inhibiting the effect of catecholamines on the $\beta$-receptors of the cardiac myocytes. The resultant decrease in inotropy and chronotropy leads to a lowering of heart rate and blood pressure. Over time, a decrease in peripheral vascular resistance is observed, although the mechanism of this decline is unclear [21]. Clinically, overdose of $\beta$-adrenergic antagonists, when taken alone, is rarely life threatening in patients whose cardiac output is not dependent on sympathetic tone. However, those patients who require high sympathetic tone, such as those with congestive heart failure, may experience serious hypotension and bradycardia as a prelude to cardiovascular collapse. $\beta$-adrenergic antagonist overdose may be accompanied by altered mental status and occasionally by hypoglycemia. The hypoglycemia is partly due to impaired glycogenolysis and gluconeogenesis.

\section{Are all calcium channel blockers associated with hypotension and bradycardia?}

There are several different types of calcium channel antagonists with differing toxicity profiles. The dihydropyridines include nifedipine, isradipine, amlodipine, nimodipine, nisoldapine and nicardipine. These agents selectively target vascular smooth muscle and predominantly cause vasodilation [22]. Toxicity from these drugs is characterized by hypotension and tachycardia, which is usually ameliorated by the administration of fluids. In more severely poisoned patients, treatment with calcium or vasopressors, such as norepinephrine, may be necessary.

Verapamil and diltiazem belong to the phenylalkylamine and benzothiazepine classes, respectively. These agents competitively inhibit L-type calcium channels on the cardiac and, to a lesser extent, vascular myocytes. In the heart, this blockade reduces the influx of calcium into the myocyte during systole and thereby decreases the dose-dependent calcium-induced calcium efflux from the sarcoplasmic reticulum. This, in turn, diminishes the excitation-contraction coupling interaction between actin and myosin, leading to decreased contractility [23]. Clinical manifestations of diltiazem or verapamil toxicity include hypotension and bradycardia because they affect the myocardial calcium channels more than the dihydropyridines [22]. Poisoning by all calcium channel blockers is often accompanied by hyperglycemia because pancreatic $\beta$-islet cells release insulin in response to calcium entry through L-type calcium channels [24].

\section{What are the options for treating patients with calcium channel antagonist toxicity?}

Patients with calcium channel blocker poisoning should be attached to a continuous ECG monitor, and IV access should be obtained with a large-bore catheter. Initiate a normal saline 
intravenous at a rapid infusion rate (based on the patient's clinical status). If the patient is hemodynamically unstable after a diltiazem or verapamil overdose, a central line should be placed with the consideration of pulmonary artery pressure monitoring to determine the relative balance of cardiac output and systemic vascular resistance. These parameters will assist with decisions about appropriate use of pressors and other pharmacotherapeutics. Orogastric lavage should be considered in patients who have ingested calcium channel blockers; however, the utility in patients who ingest sustained-release preparations is likely to be minimized by the larger size of the tablets. Caution should also be taken during lavage, given its propensity to induce bradycardia in some patients. Given the high-risk nature of calcium channel blocker poisoning, $1 \mathrm{~g} / \mathrm{kg}$ of activated charcoal (AC) should typically be administered to patients who are able to safely drink, and in some situations a nasogastric tube may be inserted for its administration. Several doses of activated charcoal $(0.5 \mathrm{~g} / \mathrm{kg})$ should be given to patients who have ingested a sustained-release product. In addition, these patients should have whole bowel irrigation (WBI) with 1-2 L/h of polyethylene glycol electrolyte lavage solution until the rectal effluent is clear.

In bradycardic patients, $0.5-1 \mathrm{mg}$ of atropine should be considered every 3-5 minutes to a maximum of $3 \mathrm{mg}(0.02 \mathrm{mg} / \mathrm{kg}$ in children). However, atropine is unlikely to be beneficial in the setting of severe toxicity, since the myocardium is globally dysfunctional in these cases and the bradycardia is not related to increased vagal tone. A potential complication of atropine administration is decreased gastrointestinal motility which can decrease the effectiveness of WBI.

Conceptually, high concentrations of extracellular calcium compete for functional channels and optimize cardiac function. In experimental animals and human cases, calcium salt administration is reported to improve hemodynamic function [25-27]. However, these effects are often transient and not always clinically effective $[28,29]$.

In cases where calcium salts do not correct hypotension, vasopressors may be required. The decision regarding vasopressor therapy should be guided by physiologic principles. Norepinephrine predominately affects $\alpha_{1}$ receptors on the vasculature, thereby causing vasoconstriction. However, the resultant increased afterload can further impair cardiac output from a poorly contractile heart. Epinephrine, at serum concentrations obtained by infusion, functions primarily as a $\beta$-agonist and has only weak $\alpha_{1}$ effects. Thus the response to epinephrine may be paradoxical, leading to improved cardiac output at the expense of peripheral vasodilation and a potential fall in the mean arterial pressure. Invasive hemodynamic monitoring is useful in aiding the decision regarding optimal use of pressors.

In animal models, myocardial glucose uptake is significantly increased in the setting of verapamil toxicity. Concomitant insulin administration enhanced glucose uptake and improved inotropy as well as survival in severely poisoned dogs [30]. Subsequent reports have shown impressive responses to insulin-glucose therapy in humans (following large overdoses with calcium channel blockers) who have exhibited refractory hypotension and bradycardia [31,32]. These successes suggest that insulin and glucose therapy should be instituted early in the course of serious calcium channel blocker toxicity and may be a useful adjunct to other pharmacologic interventions.

The aim of insulin-glucose therapy is to saturate insulin receptors and maximize cardiac glucose utilization. The dosing regimen is incompletely defined and not validated; however, an initial bolus of insulin should be about $1 \mathrm{U} / \mathrm{kg}$ accompanied by $25 \mathrm{gm}$ of dextrose (one ampule of $\mathrm{D}_{50} \mathrm{~W}$ IV) [32]. The dextrose may be withheld if the initial blood glucose is $>250 \mathrm{mg} / \mathrm{dL}$ $(13.9 \mathrm{mmol} / \mathrm{L})$. Many health care providers become uncomfortable when this dose of insulin is administered, so it should be administered in a closely monitored setting in the ED or ICU. A continuous infusion of insulin is started at $0.5 \mathrm{U} / \mathrm{kg} / \mathrm{hr}$ along with a dextrose infusion of $0.5 \mathrm{~g} / \mathrm{kg} / \mathrm{h}$ [33]. The dextrose infusion should be titrated to maintain a blood glucose concentration between 100-200 mg/dL (5.6-11.1 mmol/L).

\section{CONCLUSION}

Although it is clear that the patient reported here died from cardiotoxicity due to calcium channel blockade, it is not necessarily easy to determine the manner by which the diltiazem overdose occurred. In cases where the suspected cause of death is related to a potential toxin, a medical toxicologist can provide detailed information about the clinical pharmacology, manifestations of toxicity, and clinical management of poisoned patients to assist the Medical Examiner in determining the cause and manner of death.

\section{REFERENCES}

1. Parrish G. Assessing and improving the quality of data from medical examiners and coroners. In: Proceedings of the International Collaborative Effort on Injury Statistics: Volume I; 1994 May 18-20; Bethesda (MD): Centers for Disease Control and Prevention; 1995; $25-1$ to $25-10$.

2. Baden MM, Hennessee JA. Unnatural death: Confessions of a medical examiner. Ballantine Books. New York,1989; $58-76$.

3. Davis GG. Mind your manners: Part I: History of death certification and manner of death classification. Am J Forensic Med Pathol 1997; 18:219-223.

4. Hanzlick R. Coroner training needs: a numeric and geographic analysis. JAMA 1996; 276:1775-1778.

5. Centers for Disease Control and Prevention. http://www. cdc.gov/epo/dphsi/mecisp/summaries.htm\#FIG1. Last accessed 6 January 2006.

6. Hanzlick R, Combs D. Medical Examiner and Coroner systems: history and trends. JAMA 1998; 279:870-874.

7. Hanzlick R. Medical examiners, forensic pathologists and coroners. JAMA 1997; 277:531-532. 
8. Goodin J, Hanzlick R. Mind your manners: Part II: General results from the National Association of Medical Examiners manner of death questionnaire, 1995. Am J Forensic Med Pathol 1997; 18:224-227.

9. Hanzlick R. Lawsuits against medical examiners or coroners arising from death certificates. Am J Forensic Med Pathol 1997; 18:119-123.

10. Janofsky M: Fatal crash reveals inhalants as danger to youths: New York Times. 25 Feb 1999.

11. Baselt, RC. Disposition of Toxic Drugs and Chemicals in Man (7th edition). Foster City, CA: Biomedical Publications; 2004.

12. Package insert: Cardizem LA (Diltiazem Hydrochloride) Rev Apr 2004.

13. Moriya F, Hashimoto Y. Redistribution of diltiazem in the early postmortem period. J Anal Toxicol 2004; 28:269-271.

14. Hanzlick R. Quality assurance review of death certificates: a pilot study. Am J Forensic Med Pathol 2005; 26:63-65.

15. Preskorn SH. Fatal drug-drug interaction as a differential consideration in apparent suicides. J Psychiatr Pract 2002; 8:233-238.

16. Stephens BG Jentzen JM, Karch S, et al. National association of medical examiners position paper on the certification of cocaine-related deaths. Am J Forensic Med Pathol 2004; 25:11-13.

17. Heninger MM. Commonly encountered prescription medications in medical-legal death investigation: A guide for death investigators and medical examiners. Am J Forensic Med Pathol 2000; 21:287-299.

18. Abrams WB. In summary: Satellite symposium on central alpha-adrenergic blood pressure regulating mechanisms. Hypertension 1984; 6 (Suppl 2):87-93.

19. Van Zwieten PA. Antihypertensive drugs with a central action. Prog Pharmacol 1975; 1:1-66.

20. Bosquet P, Feldman J, Tibririca E, et al. A new concept in central regulation of the arterial blood pressure. Am J Hypertens 1992; 4:47S-50S.

21. Hoffman BB. Catecholamines, sympathomimetic drugs and adrenoceptor antagonists In Hardman JG, Limbird LE, editors. Goodman and Gilman's The Pharmacologic Basis of Therapeutics $10^{\text {th }}$ ed. New York: McGraw-Hill; 2001; 215-261.

22. Kawai C, Konishi T Matsuyama E, Okazaki H: Comparative effects of three calcium antagonists, diltiazem, verapamil and nifedipine on the sinoatrial and atrioventricular nodes. Circulation 1981; 63:1035-1042.

23. Ikemoto N: Structure and function of the calcium pump protein of the sarcoplasmic reticulum. Ann Rev Physiol 1982; 44:297-317.

24. Devis G, Somers G, Ban Obberghen E, et al. Calcium antagonists and islet function. I. Inhibition of insulin release by verapamil. Diabetes 1975; 24:547-553.

25. Hariman RJ, Mangiardi LM, McAllister RG, et al. Reversal of the cardiovascular effects of verapamil by calcium and sodium: Differences between Electrophysiologic and hemodynamic responses. Circulation 1979; 59:797-804.

26. Haddad LM. Resuscitation after nifedipine overdose occurs exclusively with intravenous calcium chloride. Am J Emerg Med 1996; 14:602-603.

27. Luscher TF, Noll G, Sturmer T, et al. Calcium gluconate in severe verapamil intoxication N Engl J Med 1994; 330: $718-720$.

28. Horowitz BZ, Rhee KJ. Massive verapamil ingestion: A report of two cases and a review of the literature. Am J Emerg Med 1989; 7:624-631.

29. Gelbke HP, Schlicht HG, Schmidt G. Fatal poisoning with verapamil. Arch Toxicol 1977; 37:89-94.

30. Kline JA, Levonas E, Raymond RM. Beneficial myocardial metabolic effects of insulin during verapamil toxicity in the anesthetized canine. Crit Care Med 1995; 23:1251-1263.

31. Boyer EW, Duic PA, Evans A. Hyperinsulinemia/euglycemia therapy for calcium channel blocker poisoning, Ped Emerg Care 2002; 18:36-37.

32. Yuan TH, Kerns WP 2nd, Tomaszewski CA, et al. Insulinglucose as adjunctive therapy for severe calcium channel antagonist poisoning. J Toxicol Clin Toxicol 1999; 37:463-474.

33. DeWitt CR, Waksman JC. Pharmacology, pathophysiology and management of calcium channel blocker and beta-blocker toxicity. Toxicol Rev 2004; 23:223-238. 